September 2013 Vol.:1, Issue:2

(c) All rights are reserved by Ong et al.

A Dieulafoy Lesion as the Cause of Massive Upper Gastrointestinal Bleeding After a Distal Pancreatectomy and

\title{
Splenectomy: Case Report and Literature Review
}

\begin{abstract}
Keywords: Upper gastrointestinal bleed; Dieulafoy lesion; Pancreatectomy

Abstract

A Dieulafoy lesion is a rare, but potentially fatal, cause of upper gastrointestinal (GI) bleeding. The literature relating to Dieulafoy lesions as a cause of GI bleeding in postoperative patients is sparse. We present the case of a 69-year-old man who developed massive hematemesis in the early postoperative period after an elective distal pancreatectomy and splenectomy, necessitating readmission to the hospital. Ultimately, the diagnosis was a Dieulafoy lesion, which was successfully treated with local injection of epinephrine, bipolar cautery, and application of hemoclips. In addition to the case report, we herein review the literature on gastric Dieulafoy lesions and highlight the importance of swift diagnosis of upper GI bleeding after pancreatic surgery.
\end{abstract}

\section{Introduction}

A Dieulafoy lesion is a rare, but potentially fatal, cause of upper gastrointestinal (GI) bleeding. Estimated to cause $1 \%$ to $2 \%$ of all GI bleeds [1-4], such lesions have been described in both adult and pediatric populations. Patients usually develop massive hematemesis, in combination with melena or hematochezia. Lesions are most commonly located in the stomach, but have been identified throughout the length of the GI tract [5-7] and more recently, in other areas such as bronchi [8]. Unless lesions are actively bleeding, they are difficult to identify and treat on initial endoscopy [9]. Treatment options include endoscopic hemostasis and endovascular interventions. Surgery was once the primary treatment, but is currently less common. After successful treatment of a patient with a Dieulafoy lesion, long-term recurrences or complications are very rare [9].

The literature relating to Dieulafoy lesions as a cause of GI bleeding in postoperative patients is sparse. Despite our extensive search of the available literature, we found only 1 other reported case of a Dieulafoy lesion in a postoperative patient. We present the case of a man who developed massive hematemesis from an upper GI bleed due to a Dieulafoy lesion, in the early postoperative period after an elective distal pancreatectomy and splenectomy. Our institutional review board exempts case reports from its review.

\section{Case Report}

The patient, a 69-year-old man with chronic pancreatitis and

\section{Journal of} Surgery

LiesI N. Close, Navaneeth C. Kumar, Evan S. Glazer, and Evan S. Ong*

Department of Surgery, University of Arizona, Tucson, AZ, USA

Address for Correspondence

Evan Ong, MD, MS, FACS, Department of Surgery, Arizona Health Sciences Center, University of Arizona, 1501 N. Campbell Ave. P.O. Box 245131, Tucson, AZ, USA 85724-5131, Tel: 520-6266664; Fax: 520-626-7785; E-mail: eong@surgery.arizona.edu

Copyright: (ㄷ) 2013 Close LN, et al. This is an open access article distributed under the Creative Commons Attribution License, which permits unrestricted use, distribution, and reproduction in any medium, provided the original work is properly cited.

Submission: 11 September 2013

Accepted: 23 September 2013

Published: 27 September 2013

Reviewed \& Approved by: Dr. Luigi Bonavina

Department of Surgery

University of Milan School of Medicine, Italy

pancreatic pseudocysts, came to our institution for an elective distal pancreatectomy and splenectomy. Two years earlier, he had undergone a laparoscopic cholecystectomy for acute gallstone pancreatitis; his intensive care unit (ICU) stay at that time was prolonged by pancreatic pseudocysts (caused by disruption and focal stricturing of his main pancreatic duct). Over the subsequent 2 years, he had been hospitalized multiple times with acute or chronic pancreatitis. A number of interventional gastroenterologists had been unable to successfully stent his pancreatic duct or perform an endoscopic gastrocystostomy, resulting in the progression of his pancreatic disease (Figure 1). Hence, we offered a distal pancreatectomy as definitive treatment; in addition, we recommended a splenectomy because of splenic vein obstruction and the associated increased risk of developing varices.

The patient tolerated the elective distal pancreatectomy and splenectomy well, with no intraoperative complications. He was transferred to our intermediate care ward for postoperative recovery. His initial course included a prolonged ileus until postoperative day 9. When he regained bowel function, he was progressively advanced to a regular diet. On postoperative day 10 he had an episode of hematemesis, with a precipitous drop in his hemoglobin level from 10.4 to $7.5 \mathrm{~g} / \mathrm{dl}$. He responded appropriately to 2 units of packed red blood cells. We attributed this isolated episode of hematemesis to a

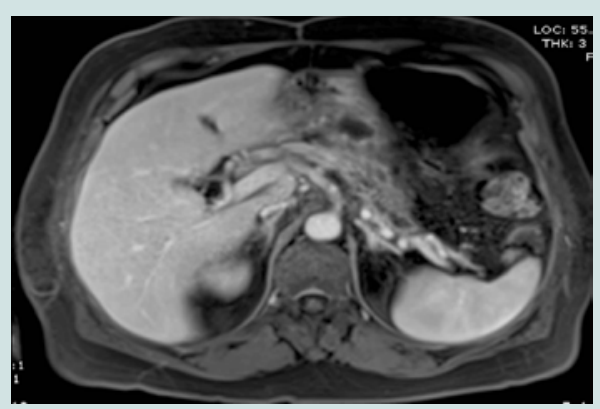

Figure 1: This preoperative T1 weighted magnetic resonance imaging (MRI) scan, with intravenous contrast, demonstrates multiple complex pancreatic pseudocysts and duct disruption in the pancreatic body and tail. 
Citation: Close LN, Kumar NC, Glazer ES, Ong ES. A Dieulafoy Lesion as the Cause of Massive Upper Gastrointestinal Bleeding After a Distal Pancreatectomy and Splenectomy: Case Report and Literature Review. J Surgery. 2013;1(2): 3.

stress ulcer. On the recommendation of the gastroenterologist, he was scheduled to undergo an upper GI endoscopy as an outpatient. On postoperative day 13 , he was discharged home in stable condition on pantoprazole.

The following day (postoperative day 14), the patient returned to the emergency department and reported melena and massive hematemesis resulting in a syncopal episode. He immediately underwent an upper GI endoscopy, which revealed "old" blood extending from the lower portion of the esophagus into the second part of the duodenum-as well as a large blood clot on the greater curvature of the stomach involving the cardia, fundus, and body. The old blood was easily washed away, but, given the perceived low probability of rebleeding, the clot was left in place. We started the patient on an intravenous pantoprazole drip and kept him on a nil per os (NPO, i.e., nothing by mouth) feeding regimen while serially measuring his hemoglobin level.

The next day (postoperative day 15), the patient continued to have hematemesis, so we decided to repeat the endoscopy and disrupt the clot. The surgical team was on standby in case of uncontrollable bleeding.

After washing the clot away, a pinpoint site of continuous ooze from a pulsating persistent vessel in the gastric fundus, with no surrounding inflammation was identified (Figure 2A). The site was then injected with a 1:10,000 solution of epinephrine for hemostasis, the lesion cauterized with a bipolar probe for tissue destruction, and 2 hemoclips placed. Meant as a precautionary measure, the hemoclips could help guide the interventional radiologist in case of rebleeding (Figure 2B).

We noted no further evidence of bleeding, either immediately after the procedure or during the remainder of his hospitalization. When the patient's laboratory test values were stable and he was able to tolerate a normal diet, he was discharged home. At follow-up, he said he had experienced no further abdominal pain, symptoms of pancreatitis, hematemesis, or melena.

\section{Discussion}

\section{Epidemiology and etiology}

To our knowledge, this is the first reported case of a Dieulafoy lesion causing upper GI bleeding after a distal pancreatectomy and splenectomy. In fact, the only other case of postoperative GI bleeding secondary to a Dieulafoy lesion that we could identify occurred in a patient after coronary artery bypass surgery [10]. Most commonly,

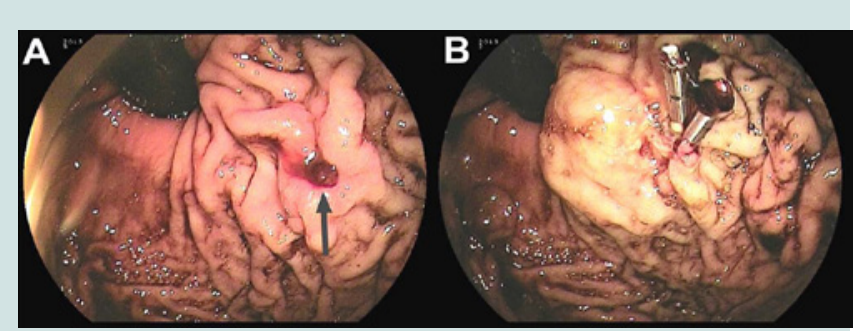

Figure 2: This pair of endoscopic photos demonstrates that, after washing away the adherent clot, we identified a small, actively oozing/bleeding vessel (A, arrow) that completely resolved with epinephrine injection, bipolar cautery, and endoscopic hemoclip placement (B).
Dieulafoy lesions have been described as a cause of GI bleeding in nonsurgical adult patients [2].

This type of lesion was initially identified in 1884 by M.T. Gallard, but was more accurately described in 1898 by Paul Georges Dieulafoy, a French physician, as a cause of fatal gastric hemorrhage. A Dieulafoy lesion occurs when an otherwise normal blood vessel maintains a constant and abnormally large diameter, failing to taper as it progresses to a capillary network in the mucosa. The diameter is typically 1 to $3 \mathrm{~mm}$ at the muscularis mucosa, nearly 10 times the normal size [11]. Running a tortuous course through the submucosa, the lesion is usually visible through a mucosal defect of 2 to $5 \mathrm{~mm}$ [11].

Dieulafoy lesions were originally described in the stomach, where more than $70 \%$ of them have been identified: the most common site has been the lesser curvature of the proximal stomach $[3,11,12]$. But they have also been found along the entirety of the GI tract, from the esophagus to the anus $[13,14]$, as well as in areas outside the GI tract, such as bronchi [8]. Histologically, intimal thickening and subintimal fibrosis have been noted at the site of the mucosal defect, but it is unclear whether those findings are part of the primary pathology or secondary to subacute bleeding. Dieulafoy lesions can be differentiated from typical gastric ulcers by the lack of inflammation and necrosis at the base surrounding the mucosal defect $[3,11]$.

The most common symptom of a Dieulafoy lesion is massive hematemesis $[3,11]$. Less common are melena, hematochezia, and a combination of those 3 symptoms. A history of peptic ulcer disease or other vascular disease has not been shown to have any correlation with Dieulafoy lesions $[3,11,13]$. Some authors have proposed that the pressure associated with the pulsations of the artery leads to erosion of the overlying mucosal epithelium, while others have hypothesized that the activity of the GI tract leads to thrombosis within the vessel, resulting in breakdown of the vessel walls $[3,4,11]$. A third theory is that age-related atrophy of the gastric mucosa leads to exposure of the underlying vessel [15].

\section{Diagnosis and treatment}

Diagnosis of an upper GI Dieulafoy lesion is typically by endoscopy [15]. Criteria for diagnosis include visualization of an active arterial bleed, of an intact vessel through a small mucosal defect with no surrounding ulceration, or of a clot that is adherent to a small mucosal defect $[3,11]$. Unfortunately, a repeat endoscopy is often necessary [9], and up to $6 \%$ of patients require 3 or more endoscopies before diagnosis $[3,11]$. The small size of the mucosal defect, the intermittent nature of the bleeding, and the reluctance to dislodge the clot all contribute greatly to delays in diagnosis.

Treatment depends not only on the location of the Dieulafoy lesion but also on the method of diagnosis. Upper GI lesions have most effectively been treated by combination therapy performed at the time of endoscopy. Initial endoscopic monotherapy is effective in $90 \%$ of patients $[2,3,11]$, yet the high rate of rebleeding favors a multimodal approach. To date, injection of a vasoconstrictor (such as epinephrine) or a sclerosing agent, and thermal coagulation have proven to be most effective $[1,9]$. Another option is mechanical treatment with endoscopic band ligation or application of hemoclips [9]. Angiography and embolization of the vessel are effective in patients who are actively bleeding or whose lesions either fail to respond to endoscopic treatment or are inaccessible by endoscope [3]. 
Citation: Close LN, Kumar NC, Glazer ES, Ong ES. A Dieulafoy Lesion as the Cause of Massive Upper Gastrointestinal Bleeding After a Distal Pancreatectomy and Splenectomy: Case Report and Literature Review. J Surgery. 2013;1(2): 3.

Surgical intervention, now considered "salvage therapy," is reserved for patients who are not helped by endoscopic or endovascular treatment.

Unfortunately, the diagnosis and treatment of our patient's Dieulafoy lesion followed a somewhat typical course, requiring multiple endoscopies for correct diagnosis. The location of his lesion in the gastric fundus made identification more difficult. The cause of the sentinel bleed during his initial hospitalization was assumed to be an ulcer or gastritis. When he returned with massive hematemesis and a large clot was visualized on endoscopy, the clot was at first left undisturbed: the perceived probability of rebleeding was less than $30 \%$; the odds were even lower, less than $10 \%$, if the problem turned out to be a simple ulcer $[16,17]$. Only after a repeat endoscopy was performed and the clot dislodged were we able to make a diagnosis and administer appropriate treatment.

At our institution, despite recent reports in the literature questioning the necessity of postoperative nasogastric tubes in pancreatic procedures [18], we routinely use such tubes in our patients until they have some evidence of return of bowel function. Nonetheless, we wonder whether the prolonged placement of the nasogastric tube in this patient irritated the mucosa over the lesion, accelerating its development.

We are unaware of any studies comparing "triple therapy" (endoscopic placement of hemoclips, epinephrine injection, and thermal ablation) as treatment for patients with a Dieulafoy lesion. But it seems quite reasonable to maximize endoscopic interventions, given the rates of morbidity and mortality associated with surgery or further episodes of massive bleeding. Some authors advocate tattooing the site of the lesion during endoscopy, in case further endoscopic or surgical interventions are needed.

\section{Conclusion}

Swift diagnosis is essential for initiating proper care and preventing catastrophic outcomes in patients with upper GI bleeding after pancreatic surgery. Although Dieulafoy lesions are uncommon, they must be included in the differential diagnosis. The reluctance to dislodge clots seems justified in the nonsurgical patient population, but we speculate that the risk/benefit ratio in the surgical population may favor more intensive interventions at the time such clots are first identified. A definitive consensus on the treatment of patients with gastric Dieulafoy lesions, including analysis of the long-term efficacy of triple therapy, would benefit the medical and surgical communities.

\section{References}

1. Chung IK, Kim EJ, Lee MS, Kim HS, Park SH, et al. (2000) Bleeding Dieulafoy's lesions and the choice of endoscopic method: comparing the hemostatic efficacy of mechanical and injection methods. Gastrointest Endosc 52: 721-724.

2. Marangoni G, Cresswell AB, Faraj W, Shaikh H, Bowles MJ (2009) An uncommon cause of life-threatening gastrointestinal bleeding: 2 synchronous Dieulafoy lesions. J Pediatr Surg 44: 441-443.

3. Baxter M, Aly EH (2010) Dieulafoy's lesion: current trends in diagnosis and management. Ann R Coll Surg Engl 92: 548-554.

4. Jamanca-Poma Y, Velasco-Guardado A, Pinero-Perez C, CalderonBegazo R, Umana-Mejia J, et al. (2012) Prognostic factors for recurrence of gastrointestinal bleeding due to Dieulafoy's lesion. World J Gastroenterol 18 : 5734-5738.

5. Baccaro L, Ogu S, Sakharpe A, Ibrahim G, Boonswang P (2012) Rectal dieulafoy lesions: a rare etiology of chronic lower gastrointestinal bleeding. Am Surg 78: E246-E248.
6. Thimmapuram J, Shah M, Srour J (2011) Esophageal Dieulafoy lesion: an unusual cause of GI bleeding. Gastrointest Endosc 73: 1055-1056.

7. Gadenstatter M, Wetscher G, Crookes PF, Mason RJ, Schwab G, et al (1998) Dieulafoy's disease of the large and small bowel. J Clin Gastroenterol 27: 169-172.

8. Parrot A, Antoine M, Khalil A, Theodore J, Mangiapan G, et al. (2008) Approach to diagnosis and pathological examination in bronchial Dieulafoy disease: a case series. Respir Res 9: 58.

9. Norton ID, Petersen BT, Sorbi D, Balm RK, Alexander GL, et al. (1999) Management and long-term prognosis of Dieulafoy lesion. Gastrointest Endosc 50: 762-767.

10. Fernandez-Sender L, Martinez-Cerezo FJ, Amoros S, Tena FJ, Marsal J, et al. (2010) Upper digestive bleeding due to Dieulafoy s lesion during the postoperative period of aortocoronary bypass surgery in a patient treated with clopidogrel. Rev Esp Enferm Dig 102: 675.

11. Lee YT, Walmsley RS, Leong RW, Sung JJ (2003) Dieulafoy's lesion. Gastrointest Endosc 58: 236-243.

12. Arora A, Mehrotra R, Patnaik PK, Pande G, Ahlawat S, et al. (1991) Dieulafoy's lesion: a rare cause of massive upper gastrointestinal haemorrhage. Trop Gastroenterol 12: 25-30.

13. Senger JL, Kanthan R (2012) The Evolution of Dieulafoy's Lesion Since 1897: Then and Now-A Journey through the Lens of a Pediatric Lesion with Literature Review. Gastroenterol Res Pract 2012: 432517.

14. Ruiz-Tovar J, Die-Trill J, Lopez-Quindos P, Rey A, Lopez-Hervas P, et al. (2008) Massive low gastrointestinal bleeding due to a Dieulafoy rectal lesion. Colorectal Dis 10: 624-625.

15. Schmulewitz N, Baillie J (2001) Dieulafoy lesions: a review of 6 years of experience at a tertiary referral center. Am J Gastroenterol 96: 1688-1694.

16. Cheng CL, Lin CH, Kuo CJ, Sung KF, Lee CS, et al. (2010) Predictors of rebleeding and mortality in patients with high-risk bleeding peptic ulcers. Dig Dis Sci 55: 2577-2583.

17. Kahi CJ, Jensen DM, Sung JJ, Bleau BL, Jung HK, et al. (2005) Endoscopic therapy versus medical therapy for bleeding peptic ulcer with adherent clot: a meta-analysis. Gastroenterology 129: 855-862.

18. Fisher WE, Hodges SE, Cruz G, Artinyan A, Silberfein EJ, et al. (2011) Routine nasogastric suction may be unnecessary after a pancreatic resection. HPB (Oxford) 13: 792-796

\section{Acknowledgements}

The authors would like to acknowledge Mary Knatterud for assistance with manuscript preparation. 